\section{The effect of}

hyaluronidase on

ultrasound-

measured dispersal

of local anaesthetic

following sub-Tenon

injection
M Khandwala'1', S Ahmed'1', S Goel'1, IG Simmons and HA McLure2
Abstract

Purpose To determine by B-scan ultrasonography if the addition of hyaluronidase affects the dispersal of anaesthetic fluid after sub-Tenon's injection.

Design Single-centre prospective randomised double-blind study.

Materials and methods We performed a trial in 19 patients who were randomised to receive $5 \mathrm{ml}$ of lidocaine $2 \%$ alone, or with hyaluronidase $15 \mathrm{IU} / \mathrm{ml}$.

A pre-anaesthetic B-scan ultrasound was performed followed by a standard infero-nasal sub-Tenon's injection. Further B-scan ultrasound studies were performed at 1,3 , and 5 min recording depth of local anaesthetic fluid. Data was analysed with Fisher's exact test and Student's $t$-test where appropriate. Results were considered significant when $P<0.05$.

Results The maximum depth of local anaesthetic was significantly less in the hyaluronidase group than the control group at 3 and $5 \mathrm{~min}(0.79$ vs $1.65 \mathrm{~mm}, P$-value 0.01 and 0.43 vs $1.52 \mathrm{~mm}, P$-value 0.002 respectively). There were no statistically significant differences in the akinesia, pain and surgical satisfaction scores between the two groups. Conclusions The addition of hyaluronidase significantly augments the dispersal of local anaesthetic fluid, as measured by B-scan ultrasonography.

Eye (2008) 22, 1065-1068; doi:10.1038/sj.eye.6702860; published online 25 May 2007

Keywords: sub-Tenon's anaesthetic; hyaluronidase; ultrasound

\section{Introduction}

Hyaluronidase is often added to the local anaesthetic solution for ophthalmic blocks. It breaks down the intercellular ground substance that binds cells together and is added to the local anaesthetic to aid dispersal of the injected solution, improving the spread and quality of block, and reducing orbital pressure. The action of hyaluronidase has been investigated extensively. Most of the data for the relatively small number of retrobulbar and sub-Tenon's studies show an improved speed of onset when hyaluronidase is used. ${ }^{1,2}$ However, there is a larger volume of studies for peribulbar anaesthesia, where the data are more conflicting. A beneficial effect has been recorded with doses of 3.75-300 IU/ml, whereas others have been unable to demonstrate a difference with doses of $0.75-150 \mathrm{IU} / \mathrm{ml}^{3-7}$ Some of this discrepancy may relate to differences in the technique, differences in the type and volume of injected local anaesthetic, and problems with the assessment of akinesia.

B-scan ultrasonography has been used to describe the distribution of local anaesthetic following a sub-Tenon injection. ${ }^{8,9}$ Following injection, a rim of fluid is seen in the retrobulbar sub-Tenon space. Over the next few minutes, this fluid rim disappears as the local anaesthetic diffuses into the surrounding tissue. We performed a study, using B-scan ultrasonography, measuring the maximum depth of the rim of fluid, to determine whether the addition of hyaluronidase affected the dispersal of local anaesthetic following sub-Tenon injection.
${ }^{1}$ Department of

Ophthalmology, St James's University Hospital, Beckett Street, Leeds, UK

${ }^{2}$ Department of Anaesthesia, St James's University Hospital, Beckett Street, Leeds, UK

Correspondence: $M$ Khandwala, 5 College Close, Lingfield, Surrey RH7 6HG, UK

Tel: + 07941604 630; Fax: +01342 837784 E-mail:mona.khandwala@ gmail.com

Received: 27 July 2006 Accepted in revised form: 13 April 2007

Published online: 25 May 2007 


\section{Materials and methods}

The study design was a prospective, double-blind, randomised, controlled trial. Approval was obtained from the local research ethics committee. Patients were eligible for inclusion if they were ASA I-III and undergoing routine cataract extraction surgery. Exclusion criteria were refusal, communication or language problems, history of allergy to amide local anaesthetic agents or hyaluronidase, known anatomical abnormalities or pre-existing extra-ocular muscle palsy. Written, informed consent was obtained from all patients. Using a computer-generated randomisation order, patients were assigned to a hyaluronidase group (group H) or a control group (group C). Group H received $5 \mathrm{ml}$ of lidocaine $2 \%$ with hyaluronidase $15 \mathrm{IU} / \mathrm{ml}$, whereas group $\mathrm{C}$ received $5 \mathrm{ml}$ of plain lidocaine $2 \%$. Syringes were prepared by an anaesthetic assistant who was not involved in the rest of the study.

Patients did not receive preoperative sedation. Standard monitoring of pulse-oximetry and ECG were used. An intravenous cannula was placed. Globe and eyelid movements were assessed using the scoring system in Table 1. A pre-anaesthetic B-scan ultrasound was performed to check for normal anatomy. Topical anaesthesia of conjunctiva and cornea was achieved by administering 2-3 drops of proxymetacaine $0.5 \%$ followed by tetracaine $1 \%$ drops. Iodine drops were applied to the conjunctival fornix and around the eyelids, followed by a standard infero-nasal sub-Tenon's injection, as described by Stevens (Visitec 19G $25 \mathrm{~mm}$ sub-Tenon cannula). ${ }^{10}$ No compression of the eye was performed. All patients had a sub-Tenon's block and no sub-conjunctival fluid was seen in any of the patients. Further B-scan ultrasound studies were performed at 1, 3 , and $5 \mathrm{~min}$. For the measurement of the depth of anaesthetic fluid, we used the B-scan ultrasound probe in an axial position to first identify the optic nerve. Once the anaesthetic fluid was located, we directed the ultrasound probe to identify the maximal depth of fluid to be measured. Measurements were taken from the frozen image using the available function from the B-scan hardware. To ensure standardization, the same procedure was followed in all cases and all scans were performed by the same ophthalmologist. At each scan,

Table 1 Akinesia scoring system

\begin{tabular}{lc}
\hline Movement & Score \\
\hline Full & 3 \\
Partial & 2 \\
Flicker & 1 \\
No movement & 0 \\
\hline
\end{tabular}

the maximum depth of local anaesthetic was located and measured. All blocks were performed by one anaesthetist (HM), and all ultrasound scans were performed by one ophthalmologist (MK), both of whom were blinded to which group the patients were allocated. At each scan, the maximum depth of local anaesthetic was located, measured and recorded. After the last ultrasound, ocular movements were assessed. Time from the first injection to start of surgery and duration of surgery was recorded. At completion of surgery, the surgeon was asked to score the surgical conditions using a verbal analogue scale (VAS) from 0 (worst) to 10 (best) and the patients were asked to score pain using a VAS from 0 (no pain) to 10 (worst imaginable). Data between groups were compared using Student's $t$-test and Fisher's exact test where appropriate. A $P$-value of $<0.05$ was considered significant.

\section{Results}

Twenty patients were enrolled in the study. However, data for one patient in group $\mathrm{H}$ were incomplete and were excluded from analysis. Demographic data were comparable between group $\mathrm{H}$ and group $\mathrm{C}$ with respect to mean age (73.8 vs 74.0 years, $P$-value 0.98$)$, ASA classification ( $P$-value 0.28$)$ and axial length $(23.6$ vs $23.5 \mathrm{~mm}, P$-value 0.94$)$. The maximum depth of local anaesthetic was significantly less in the hyaluronidase group than the control group at 3 and $5 \mathrm{~min}$, with a nonsignificant trend at $1 \mathrm{~min}$ (Figure 1). There were no statistically significant differences between group $\mathrm{H}$ and group $C$ in terms of mean akinesia scores at $5 \mathrm{~min}$ (2.6 vs 3.0, $P$-value 0.65 ), pain scores (1.4 vs $0.7, P$-value $0.5)$ or surgical satisfaction scores (9.2 vs $9.2, P$-value 0.96$)$.

\section{Discussion}

Hyaluronidase may be added to the local anaesthetic solution to aid dispersal, improving the onset and quality of block, and 'softening' the eye to optimise the surgical field. In the past, researchers investigating the efficacy of hyaluronidase have provided mixed results. This discord

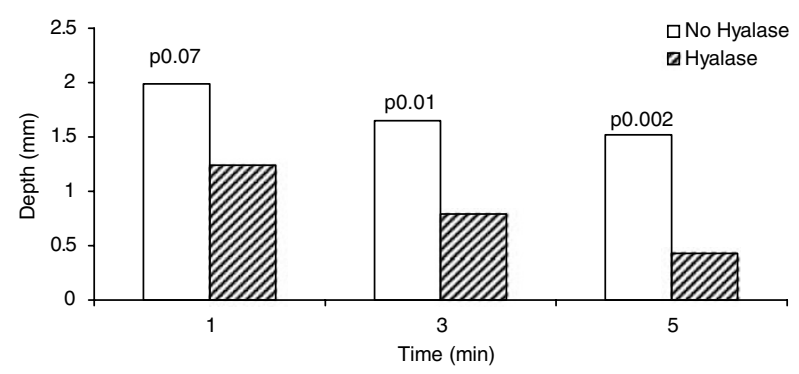

Figure 1 Depth of fluid. 
may be a result of focus on the impact of hyaluronidase on ocular akinesia. Although a superficially attractive outcome to measure, the accurate assessment of akinesia is difficult. Studies are often performed using the elderly cataract population, who may not hear or understand instructions, or who may tire rapidly if the study is intensive or prolonged. There is no uniformly agreed method of assessing akinesia, and a wide variety of different scales have been used, all of which are subjective and open to observer error. In addition, although complete akinesia used to be an essential endpoint of ophthalmic block, surgical techniques have evolved and an immobile eye is no longer an absolute requirement for most surgeons. Our study aimed to look at the effect of hyaluronidase on dispersal of local anaesthetic, which may have significant consequences for the surgical field and visual outcome. We used assessment of the change in depth of fluid in the sub-Tenon space as a marker of dispersal of local anaesthetic. The precise location around the globe where the measurement was performed could not be standardised as even with the same injection site and technique of injection, the fluid distributes differently between individuals. Instead, we relied on measuring the maximum depth that could be visualised, and noted the change in depth over a period of time (Figure 2). The depth of local anaesthetic did not appear to increase between scans in any of the patients.

Our study showed that the addition of hyaluronidase appeared to improve the dispersal of local anaesthetic fluid away from the sub-Tenon space. An improvement in akinesia or analgesia might have been anticipated, but this was not seen. This is unsurprising as we measured akinesia after $5 \mathrm{~min}$. Lidocaine $2 \%$ is a rapidly acting local anaesthetic with a mean onset time of 3 min when used with hyaluronidase for sub-Tenon anaesthesia. ${ }^{11}$ The delay in assessing akinesia till 5 min after the injection may have masked a significant difference between the groups. In addition, ours was a small study, inadequately powered to detect small differences in akinesia with the use of hyaluronidase. Similarly, there was no difference in pain scores. Indeed, there is no evidence that hyaluronidase significantly improves the analgesic effects of local anaesthetics for ophthalmic surgery. Our surgeons could find no difference in the quality of the surgical field between the groups. There are little data on the effects of hyaluronidase on the surgical field, although some improvement in the surgical field and reduction in complications with the use of hyaluronidase has been recorded. ${ }^{12}$ It is likely that a much larger study would be required to establish whether hyaluronidase has a statistically significant impact on the surgical field.

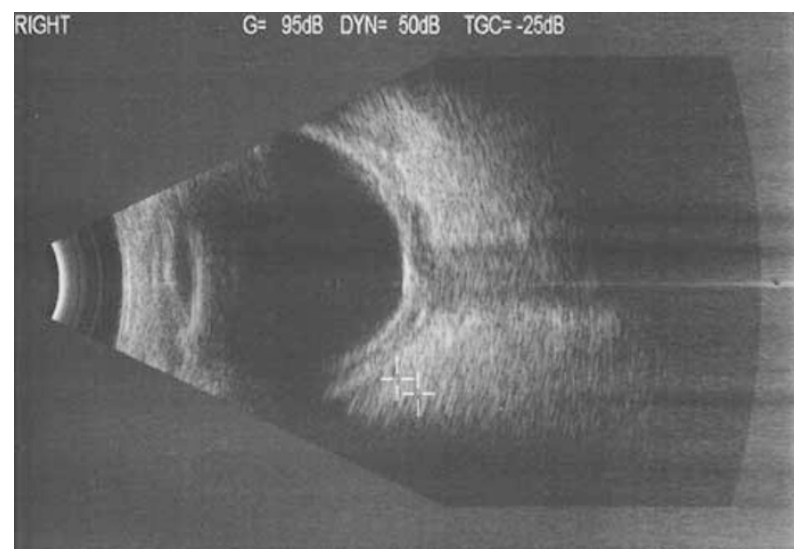

Figure 2 Ultrasound showing the characteristic ' $\mathrm{T}$ ' sign after sub-Tenon's anaesthetic with hyaluronidase.

Hyaluronidase may offer improvements in akinesia, although this is a diminishing requirement for many ophthalmic surgeons. It appears to offer little other measurable improvement in block for the patient or surgeon, and it is an additional and avoidable expense, with documented allergic problems. ${ }^{13}$ The continued use of hyaluronidase might seem unjustified. However, supporting evidence for hyaluronidase is emerging from periods when hyaluronidase was unavailable. In the United States, shortages of hyaluronidase in 1998 and 2000 were followed by clusters of post-cataract extraction diplopia requiring surgical correction. ${ }^{14,15}$ A similar pattern of muscle damage was seen in France when hyaluronidase was withdrawn by the Agency for Health Product Safety in $2001 .^{16}$ This seems to indicate a protective effect of hyaluronidase. Local anaesthetics are known to be myotoxic. Direct injection into muscle provokes an intense inflammatory reaction, which may progress to necrosis, following by healing with fibrosis and retraction. In the delicate extraocular muscles this may result in a significant muscle injury requiring surgical correction. Hyaluronidase encourages dispersal of local anaesthetic, reducing the exposure of individual extraocular muscles to local anaesthetic, which may reduce the incidence of muscular injury. In sub-Tenon's anaesthesia, a blunt rather than sharp needle is inserted, so direct intramuscular injection should be less likely. However, muscular injury following sub-Tenon's injection has been reported and may be caused by local anaesthetic seeping around the muscle through fenestrations in the Tenon's fascia. ${ }^{17}$ Consequently, hyaluronidase may have a protective action even with non-sharp needle techniques. Our study gave us a visual confirmation that solutions containing hyaluronidase resulted in more rapid dispersal of local anaesthetic, which may protect delicate ocular muscles and improve the surgical field. 


\section{Acknowledgements}

This study did not receive any funding from private or public bodies. The authors state no conflict of interest.

\section{References}

1 Nicoll J, Treuren B, Acharya PA, Ahlen K, James M. Retrobulbar anesthesia: the role of hyaluronidase. Anaesth Analg 1986; 65: 1324-1328.

2 Guise P, Laurent S. Sub-Tenon's block: the effect of hyaluronidase on speed of onset and block quality. Anaes Intensive Care 1999; 27: 179-181.

3 Kallio H, Paloheimo M, Maunuksela E. Hyaluronidase as an adjunct in bupivacaine-lidocaine mixture for retrobulbar/ peribulbar block. Anaesth Analg 2000; 91: 934-937.

4 Brydon C, Basler M, Kerr W. An evaluation of two concentrations of hyaluronidase for supplementation of peribulbar anaesthesia. Anaesthesia 1995; 50: 998-1000.

5 DeBroff B, Hamilton R, Loken R, Gimbel HV, Ferensowicz M. Retrobulbar anaesthesia with 7.5 vs $0.75 \mathrm{IU} / \mathrm{ml}$ of hyaluronidase. Can J Ophthalmol 1995; 30: 262-264.

6 Dempsey G, Barrett P, Kirby I. Hyaluronidase and peribulbar block. Br J Anaesth 1997; 78: 671-674.

7 Bowman R, Newman DK, Richardson EC, Callear AB, Flanagan DW. Is hyaluronidase helpful for peribulbar anaesthesia? Eye 1997; 11: 385-388.

8 Kumar C, McNeela B. Ultrasonic localization of anaesthetic fluid using sub-Tenon's cannula of three different lengths. Eye 2003; 17: 1003-1007.
9 Winder S, Walker S, Atta H. Ultrasonic localization of anaesthetic fluid in sub-Tenon's, peribulbar and retrobulbar techniques. J Cataract Refract Surg 1999; 25: 56-59.

10 Stevens J. A new local anaesthesia technique for cataract extraction by one quadrant sub-Tenon's infiltration. $\mathrm{Br} \mathrm{J}$ Ophthalmol 1992; 76: 670-674.

11 McLure H, Patel A, Kumar C. A comparison of lidocaine 2\% with levobupivacaine $0.75 \%$ for sub-Tenon's anaesthesia. Eur I Anaesthesiol 2005; 22: 500-503.

12 Krohn J, Seland JH, Hovding G, Aasved H. Retrobulbar anesthesia with and without hyaluronidase in extracapsular cataract surgery: a prospective, randomized, double-blind study. Acta Ophthalmol Scand Suppl 1993; 71: 791-795.

13 Agrawal A, McLure H, Dabbs T. Hyaluronidase allergy following peribulbar anaesthesia. Anaesthesia 2003; 58: 493-494.

14 Brown S, Brooks SE, Mazow ML, Avilla CW, Braverman DE, Greenhaw ST. Cluster of diplopia cases after periocular anaesthesia without hyaluronidase. J Cataract Refract Surg 1999; 25: 1245-1249.

15 Brown S, Coats DK, Collins ML, Underdahl JP. Second cluster of strabismus cases after periocular anaesthesia without hyaluronidase. J Cataract Refract Surg 2001; 27: 1872-1875.

16 Jehan F, Hagan III JC, Whittaker TJ, Subramanian M. Diplopia and ptosis following injection of local anaesthesia without hyaluronidase. J Cataract Refract Surg 2001; 27: 1876-1879.

17 Adams W, Morgan S. Diplopia following sub-Tenon's infiltration of local anaesthesia. J Cataract Refract Surg 2002; 28: 1694-1697. 